\title{
Methylated-CpG island recovery assay: a new technique for the rapid detection of methylated-CpG islands in cancer
}

\author{
Tibor Rauch and Gerd P Pfeifer
}

Division of Biology, Beckman Research Institute of the City of Hope, Duarte, CA, USA

\begin{abstract}
Hypermethylation of CpG islands is a phenomenon commonly observed during the development and progression of human tumors. Detection of methylated-CpG islands in easily accessible biological materials such as serum has the potential to be useful for the early diagnosis of cancer. Most currently used methods for detecting methylated-CpG islands are based on sodium bisulfite conversion of genomic DNA, followed by PCR reactions. Here we describe a method, methylated-CpG island recovery assay (MIRA) that does not depend on the use of sodium bisulfite but has similar sensitivity and specificity as bisulfite-based approaches. MethylCpG-binding domain proteins, such as methyl-CpG-binding domain protein-2 (MBD2), have the capacity to bind specifically to methylated DNA sequences. In the MIRA procedure, sonicated genomic DNA isolated from cells or tissue is incubated with a matrix containing glutathione-S-transferase-MBD2b in the presence of methylCpG-binding domain protein 3-like-1, a binding partner of MBD2 that increases the affinity of MBD2 for methylated DNA. Specifically bound DNA is eluted from the matrix and gene-specific PCR reactions are performed to detect $\mathrm{CpG}$ island methylation. Methylation can be detected using $1 \mathrm{ng}$ of DNA or 3000 cells. MIRA is a specific and sensitive, but not laborious, technique that can be clinically useful in the detection and diagnosis of any DNA methylation-associated disease, including cancer.

Laboratory Investigation (2005) 85, 1172-1180. doi:10.1038/labinvest.3700311; published online 18 July 2005
\end{abstract}

Keywords: DNA methylation; methyl-CpG-binding domain protein; MBD2; RASSF1A; GSTP1; cancer diagnosis

Methylation of DNA at CpG dinucleotides is one of the most important epigenetic modifications in mammalian cells. Short regions of DNA in which the frequency of $5^{\prime}-\mathrm{CG}-3^{\prime}(\mathrm{CpG})$ dinucleotides is higher than in other regions of the genome are called CpG islands. ${ }^{1} \mathrm{CpG}$ islands often harbor the promoters of genes and play a pivotal role in the control of gene expression. In normal tissues CpG islands are usually unmethylated, but a subset of islands becomes methylated during tumor development. $^{2-4}$ Methyl-CpG-binding domain (MBD) proteins specifically recognize methylated DNA sequences and are essential components of regulatory complexes that mediate transcriptional repression of methylated DNA., ${ }^{5,6}$ One of the bestcharacterized members of the MBD protein family is the methyl-CpG-binding domain protein-2 (MBD2). MBD2 has two isoforms, MBD2a and

Correspondence: Dr GP Pfeifer, PhD, Division of Biology, City of Hope Cancer Center, 1500 E Duarte Road, Duarte, CA 91010, USA. E-mail: gpfeifer@coh.org

Received 26 March 2005; revised and accepted 18 May 2005; published online 18 July 2005
MBD2b, which are alternatively translated from the same mRNA. ${ }^{7}$ Recent studies indicate that interacting proteins can modulate the methylated DNA-binding ability of the MBD2 protein. ${ }^{8}$ MethylCpG-binding domain protein 3-like-1 (MBD3L1) interacts with $\mathrm{MBD} 2 \mathrm{~b}$ in vivo and in vitro and promotes the formation of methylated-DNA-binding complexes. ${ }^{8}$

In order to identify and characterize the chromosomal regions (particularly CpG islands) that undergo de novo methyation in tumorigenesis, many technical approaches have been used..$^{3,9,10}$ These methods can be classified into several groups on the basis of their principles. The first group of techniques is based on restriction endonuclease cleavage. These techniques require the presence of methylated cytosine residues within the recognition sequence that affect the cleavage activity of restriction endonucleases (eg, HpaII, HhaI). ${ }^{11}$ Methylation of the genomic target can be detected by Southern blot hybridization. The technique is limited to specific restriction sites and requires large amounts of DNA. The second set of techniques makes use of the differential sensitivity of cytosine and 5-methyl- 
cytosine towards chemical modification and cleavage by employing Maxam-Gilbert sequencing technology. ${ }^{12}$ The application of ligation-mediated PCR techniques to Maxam-Gilbert treated genomic DNA allows the exact identification and partial quantification of 5-methylcytosines at the singlenucleotide level in mammalian genes. ${ }^{13}$ Although highly specific and reasonably sensitive (requires 0.5-1 $\mathrm{g}$ of DNA), these techniques are technically complex. The principle of bisulfite genomic sequencing is that methylated and unmethylated cytosine residues react in a different manner with sodium bisulfite. ${ }^{14,15}$ After bisulfite treatment of genomic DNA, the unmethylated cytosines are converted to uracils by deamination, while methylated cytosine residues can hardly react with this agent and remain intact. After this chemical treatment the region of interest must be PCR amplified, and in most cases cloned and sequenced. Alignment analysis of the original (untreated) and cloned (treated) nucleotide sequences can reveal the in vivo methylation status of the amplified region. The PCR products obtained from bisulfite-treated DNA can also be analyzed by combined bisulfite-restriction analysis (COBRA assay), which can distinguish between methylated and unmethylated DNA. ${ }^{16}$ Another commonly used sodium bilsulfite-dependent technique is methylation-specific PCR (MSP). ${ }^{17}$ Sodium bisulfite-treated genomic DNA serves as the template for a subsequent PCR reaction. Specific sets of PCR primers are designed in such a way to discriminate between bisulfite-modified and unmodified template DNA, and between unmethylated (deaminated) and methylated (nondeaminated) cytosines at CpG sites. Another approach used for the identification of methylated-CpG islands utilizes the ability of the MBD domain of the MeCP2 protein to selectively bind to methylated DNA sequences. ${ }^{18,19}$ The bacterially expressed and purified His-tagged methylCpG-binding domain is immobilized to a solid matrix and used for preparative column chromatography to isolate highly methylated DNA sequences. Restriction endonuclease-digested genomic DNA is loaded onto the affinity column and methylatedCpG island-enriched fractions are eluted by a linear gradient of sodium chloride. PCR or Southern hybridization techniques are used to detect specific sequences in these fractions. There are several additional methods for analysis of methylation patterns, but each of them is a derivative of the above-mentioned principles. ${ }^{9,10}$

Here we report on the development of a new in vitro method to investigate the methylation status of CpG islands. The methyl-CpG island recovery assay (MIRA) is based on the fact that the MBD2b protein can specifically recognize methylated-CpG dinucleotides and this interaction is enhanced by the MBD3L1 protein. ${ }^{8}$ Matrixassisted binding and simple PCR assays are used to detect methylated DNA sequences in the recovered fraction.

\section{Materials and methods}

\section{Plasmid Constructs and Protein Expression}

For the expression of glutathione- $S$-transferase (GST)-tagged recombinant MBD2b protein, the RT-PCR-generated full-length cDNA was cloned into the pGEX-5X-1 expression vector (Amersham Pharmacia Biotech). The His-tagged MBD3L1 protein expression vector was constructed as described earlier. ${ }^{8}$ Expression constructs were transformed into Escherichia coli BL21(DE) and expression was induced with $0.5 \mathrm{mM}$ IPTG for $4 \mathrm{~h}$ at $37^{\circ} \mathrm{C}$. Bacterial pellets were resuspended in ice-cold STE buffer (10 mM Tris-HCl, $\mathrm{pH}$ 7.8, $150 \mathrm{mM} \mathrm{NaCl}$, $1 \mathrm{mM}$ EDTA) containing $100 \mu \mathrm{g} / \mathrm{ml}$ lysozyme. After $10 \mathrm{~min}$ incubation on ice, bacteria were lysed by addition of $1.5 \%$ of $N$-lauroylsarcosine (final concentration) and sonicated three times for $1 \mathrm{~min}$. The lysate was cleared by centrifugation and Glutathione Sepharose 4B (Amersham Bioscience), or Ni-NTA His-Bind ${ }^{\circledR}$ Resin (Novagen) was added. Glutathione beads were washed three times with ice-cold PBS and resuspended in storage buffer $(50 \mathrm{mM}$ Hepes, $\mathrm{pH} 7.4,150 \mathrm{mM} \mathrm{NaCl}, 5 \mathrm{mM}$ DTT, $10 \%$ glycerol). Solid matrix-bound MBD2b was kept at $4^{\circ} \mathrm{C}$ for several weeks. Ni-NTA beads were washed and His-tagged MBD3L1 eluted according to the manufacturer's recommendations (Novagen). Eluted MBD3L1 was dialyzed against storage buffer ( $50 \mathrm{mM}$ Hepes, pH 7.4, $150 \mathrm{mM} \mathrm{NaCl}, 5 \mathrm{mM}$ DTT, $50 \%$ glycerol) and kept at $-20^{\circ} \mathrm{C}$. Purity and concentrations of the recombinant proteins were determined by SDS-polyacrylamide gel (PAGE) and staining with Coomassie blue.

\section{Cell Culture and DNA Purification}

DNA samples were obtained from pancreatic and prostate cancer tissues and from normal blood sample controls. A549 cells were obtained from the American Type Culture Collection (Manassas, VA, USA). The prostate epithelial cell lines 267B1 and $\mathrm{Ki} / \mathrm{HPV}$ were kindly provided by Johng Rhim (Uniformed Services University of the Health Sciences, Bethesda, MD, USA). Mammalian cells were cultured in DMEM supplemented with 10\% fetal bovine serum. Genomic DNA was purified from eukaryotic cells by a standard procedure using phenol chloroform extraction and ethanol precipitation. When the MIRA procedure was started directly from human cells, adherent cells were washed with cold PBS and scraped by using a rubber policeman. Scraped cells were centrifuged at $1000 \mathrm{~g}$ for $2 \mathrm{~min}$ at $4{ }^{\circ} \mathrm{C}$ and the cell pellet was resuspended in guanidinium hydrochloride-containing binding buffer (Qiaquick PCR purification kit; Qiagen). Genomic DNA was sonicated in this solution to an average fragment length of $0.35 \mathrm{~kb}$ (corresponding to an average mass distribution of $0.7 \mathrm{~kb}$ on an ethidiumstained gel) and loaded onto the positively charged 
purification column (Qiaquick PCR purification kit; Qiagen). The bound fragment population was eluted according to the manufacturer's recommendations.

\section{Methylated-CpG Island Recovery Assay (MIRA)}

GST protein $(1 \mu \mathrm{g})$ or GST-tagged MBD2b protein bound to Glutathione Sepharose CL-4B matrix was incubated with sonicated and purified genomic DNA in the binding reaction mixture $(10 \mathrm{mM}$ Tris$\mathrm{HCl}, \mathrm{pH}$ 7.5, $50 \mathrm{mM} \mathrm{NaCl}, 1 \mathrm{mM}$ EDTA, $1 \mathrm{mM}$ DTT, $3 \mathrm{mM} \mathrm{MgCl}{ }_{2}, 0.1 \%$ Triton-X100, $5 \%$ glycerol and $25 \mu \mathrm{g} / \mathrm{ml} \mathrm{BSA}$ ) for $20 \mathrm{~min}$ at $4^{\circ} \mathrm{C}$ on a rocking platform. After washing the pelletted Sepharose beads three times with binding buffer containing $700 \mathrm{mM} \mathrm{NaCl}$, the methylated DNA-enriched genomic DNA fraction was eluted by addition of guanidinium hydrochloride-containing buffer and purified using Qiaquick PCR purification kits (Qiagen). When His-tagged MBD3L1 was added to the binding reaction, a pre-incubation step $(20 \mathrm{~min}$ at $4^{\circ} \mathrm{C}$ on a rocking platform) was introduced before adding the sonicated genomic DNA. In all, $1 \mu \mathrm{g}$ of purified His-tagged MBD3L1 protein was added for the pre-incubation step.

\section{PCR of CpG Islands}

The M35 and M5L Ras association domain family 1A gene $(R A S S F 1 A)$ promoter fragment was amplified by using 20 pmol of each primer: $5^{\prime}$-GCCAAGTGTGTTGCTTCAGCAAACCG-3' ${ }^{\prime}$ and $5^{\prime}$ CCCGCAGCTCAATGAGCTCAGGCT- ${ }^{\prime}$. For generation of the M1 and M10 fragments, the following primer pair was used: 5'-ATCGCCAAGTGTGTTGC TTCAGCAA- $3^{\prime}$ and $5^{\prime}$-ATCCAAAGCACCACGC-GG AGATA-3', while for detection of M2 and M5S, 5'-ATC TTCCTCTAGCACAGTAAAGCTGGCC- $3^{\prime}$ and $5^{\prime}$-ATC CTTCCCTCC-TTCGTCCCCTCC- $3^{\prime}$ oligonucleotides were used. For M3 amplification, 5'-ATCGC CAAGTGTGTTGCTTCAGCAA- $3^{\prime}$ and $5^{\prime}$-ATCCTTC CCTCCTTCGTC-CCCTCC-3' ${ }^{\prime}$ primers were applied. For LINE-1 promoter detection, the following primer pair was used: 5'-GCTCCGGTCTACAGCTCCCAGC GT-3' and $5^{\prime}$-AGCTGTGGTGGGCTCC-ACCCAGTT$3^{\prime}$. For amplification of the GSTP1 promoter after enrichment of methylated DNA by MIRA, the following primers were used: $5^{\prime}$-CCCGGGGTGCAG CGGCCGCC- $3^{\prime}$ and $5^{\prime}$-GCCCCAGTGCTGAGTCACG GCG-3'. The PCR reactions were performed according to the manufacturer's recommendations (Qiagen) using Q solution and 1.25 U of HotStart Taq enzyme per reaction. PCR cycling conditions were $92^{\circ} \mathrm{C}$ for $20 \mathrm{~s}, 60^{\circ} \mathrm{C}$ for $30 \mathrm{~s}$ and $72^{\circ} \mathrm{C}$ for $30 \mathrm{~s}$. The optimal PCR cycle number was varied, but for starting the analysis an initial 28 cycles are suggested. The PCR products were separated by agarose gel electrophoresis. PCR should be performed in such a way that cycling is stopped while the reaction is still in the linear stage of amplification.

\section{Testing of the Binding Capacity of the MIRA Matrix}

MIRA analysis was performed on a total of $500 \mathrm{ng}$ of input DNA after mixing 375, 250, 125 and $0 \mathrm{ng}$ of A549 DNA with 0, 125, 250 and $375 \mathrm{ng}$ of HeLa DNA, respectively. Supernatant fractions were concentrated using Qiaquick column (QIAGEN) according to the manufacturer's instructions. In all, $1 \mu \mathrm{g}$ of sonicated herring sperm DNA was added to the isolated DNA samples and these were treated with sodium bisulfite. ${ }^{17}$ The bisulfite-modified templates were monitored by MSP. For MSP analysis of the RASSF1A promoter, the following primer pairs were used: METFor- $-5^{\prime}$-GGGTTTTGCGAGAGCGCG-3' ${ }^{\prime}$ and METRev-5'-GCCCCAATACTAAAT-CACGAC G-3'; UNMFor-5'-GGGGTTTTGTGAGAGTGTGTTTAG-3' and UNMRev-5'-TAAACACTAACAAACACAAAC CAAAC- $3^{\prime}$.

\section{Bisulfite Sequencing Analysis}

MIRA was performed on pre-mixed $250 \mathrm{ng}$ of A549 DNA (methylated RASSF1A promoter) and $250 \mathrm{ng}$ of HeLa DNA (unmethylated RASSF1A promoter). DNA was purified from the supernatant and the pellet fraction. In all, $1 \mu \mathrm{g}$ of sonicated herring sperm DNA was added and sodium bisulfite treatment was performed on these samples. The RASSF1A promoter was amplified by semi-nested PCR. In the first PCR, we used primer MU379 (5'-GTTTTGGTAGTT TAATGAGTTTAGGTTTTTT-3') and primer ML370 (5'-ACCCTCTTCCTCTAACACAATAAAACTAACC$\left.3^{\prime}\right)$. These primers amplify both unmethylated and methylated bisulfite-converted DNA templates. $1 / 50$ volume of the first reaction was used as input in the second round of PCR. The internal primer MU561 (5'-CCCCACAATCCCTACACCCAAAT-3') and the MU379 primers were used for amplification. The PCR products were cloned into the pDrive cloning vector (Qiagen) and 10 randomly selected recombinant clones were sequenced from the supernatant and pellet fraction, respectively.

\section{Testing of the Effect of Methylation Density on MIRA}

PCR-amplified and purified RASSF1A promoter fragments were in vitro methylated with either SssI or HpaII methyltranferase to obtain different methylation densities (New England Biolabs). In all, $1 \mathrm{ng}$ of the artificially methylated promoter and $100 \mathrm{ng}$ of sonicated JM110 bacterial DNA were added to the binding reaction in the MIRA assay. Appearance of the methylation-specific signal in the bound fraction was analyzed after 20,25 and 30 PCR cycles.

\section{Results and discussion}

The MIRA was developed as a GST pull-down method in which bacterially expressed and solidphase-bound recombinant MBD2b protein is incu- 
bated with sonicated total genomic DNA. After washing of the beads with high-salt buffer and elution of bound DNA, a gene-specific PCR reaction is performed on the isolated fragments to detect the recovered $\mathrm{CpG}$ islands (Figure 1).

From recently published studies, it is known that MBD2b possesses one of the highest affinities for methylated DNA among the MBD proteins. ${ }^{20}$ MBD2b interacts with MBD3L1 and this interaction further strengthens MBD2b's binding affinity for methylated templates. ${ }^{8}$ The MBD3L1 interaction domain was mapped to the C-terminal end of MBD2b and therefore we used the full-length MBD2b protein and not just the MBD domain in the MIRA procedure. Column chromatography has already proven that the MBD of another MBD protein, MeCP2, immobilized to a solid matrix is suitable for selective retention of highly methylated-CpG islands. ${ }^{18,19}$ The tumor suppressor gene RASSF1A was cloned and characterized in our laboratory, ${ }^{21}$ and it has been shown that its promoter is frequently methylated in many human cancer cell lines and primary tumors. ${ }^{22}$

We first bacterially expressed and purified the recombinant proteins used in the subsequent experiments (Figure 2a). Purified proteins were kept on the surface of Glutathione Sepharose beads at $4^{\circ} \mathrm{C}$ until used. We next sought to define the sensitivity of MIRA for detection of the methylated RASSF1A promoter. From a previous study using bisulfite sequencing, it is known that the $R A S S F 1 A$ promoter is methylated in the A549 lung cancer cell line. ${ }^{21}$ Sonicated DNA from the A549 cell line was mixed with solid-phase-bound GST-MBD2b and, as a negative control, with matrix-bound GST protein. Decreasing amounts of genomic DNA $(1,0.5,0.25$, $0.125,0.062,0.031,0.025,0.01$ and $0.001 \mu \mathrm{g})$ were added in the titration experiment. We could detect a positive signal for methylated DNA in the sample, in

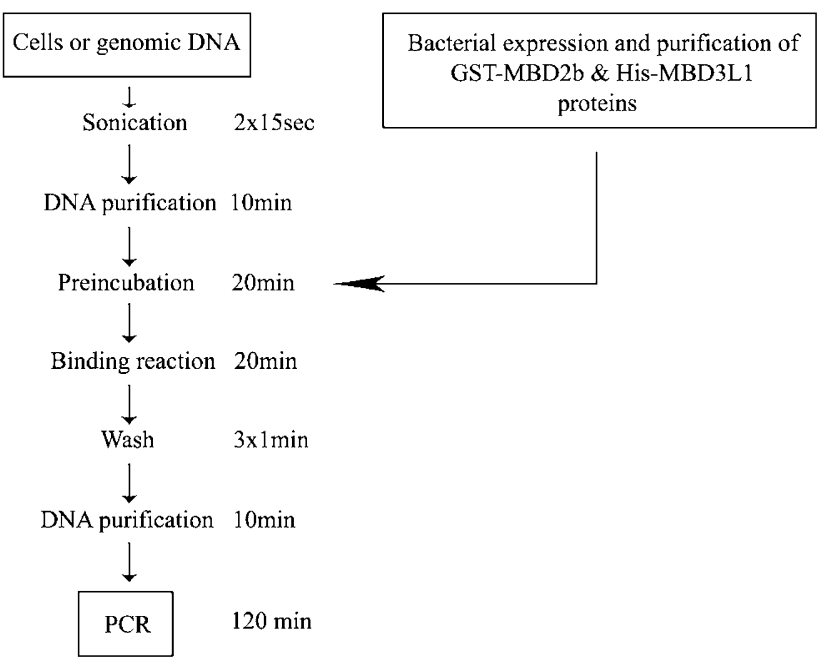

Figure 1 Schematic diagram of the MIRA. which $\sim 62$ ng of DNA was added (Figure 2b, lane 8). No signal was detected in samples containing only GST protein (Figure $2 \mathrm{~b}$, lane 3 ), although the input DNA was the highest $(1 \mu \mathrm{g})$.

MBD3L1 has significant homology to MBD2 and MBD3. Its MBD is missing and the protein cannot bind to methylated DNA alone. ${ }^{23}$ Recent in vitro binding experiments have shown that MBD3L1 can

a

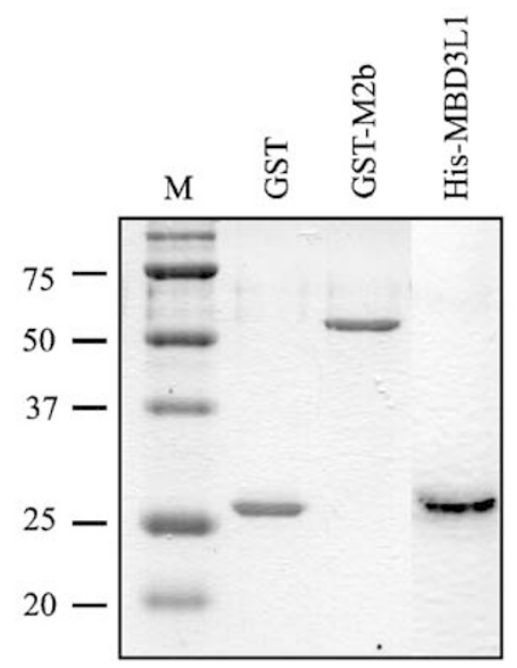

b Sonicated genomic DNA $1000 \mathrm{ng}$

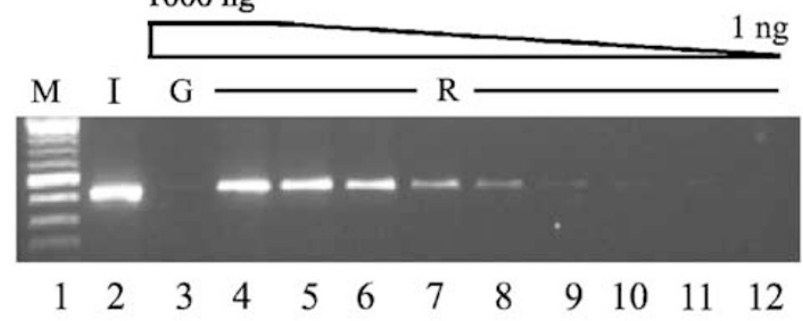

c Sonicated genomic DNA

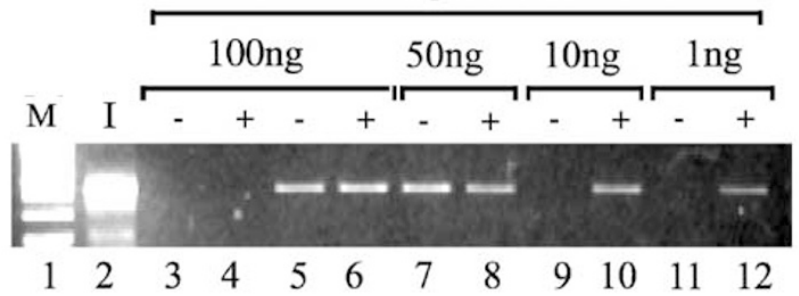

Figure 2 Determination of the sensitivity of MIRA. (a) Purified proteins used in the assay are separated on a 13\% SDS-PAGE. (b) Decreasing amounts of sonicated genomic DNA $(1,0.5,0.25$, $0.125,0.062,0.031,0.025,0.01$ and $0.001 \mu \mathrm{g}$ ) isolated from A549 cells were incubated with GST-fused MBD2b protein. 'I' represents the input genomic DNA and 'R' stands for the recovered samples. In the negative control reaction, GST protein only (' $G$ ') was incubated with $1 \mu \mathrm{g}$ of sonicated DNA. PCR was performed with primers specific for the RASSF1A promoter. (c) The assay was performed both in the absence $(-)$ and in the presence $(+)$ of MBD3L1 protein. 'I' stands for input DNA. Lanes 3 and 4, GST protein only; lanes 5-12, GST-MBD2b. 
interact with MBD2b and can enhance methylatedDNA-specific complex formation in a dose-dependent manner. ${ }^{8}$ To test whether the MBD3L1 protein's positive effect on MBD2b-methylated DNA complex formation can improve the sensitivity of the MIRA assay, we pre-incubated matrix-bound GST-MBD2b with MBD3L1 and compared the detection efficiency with that of reactions performed without MBD3L1 (Figure 2c). We found that MBD3L1 could increase the sensitivity of the assay and as low as $1 \mathrm{ng}$ of input A549 DNA was sufficient to detect the methylated RASSF1A promoter (Figure 2c, lane 12). The positive effect of MBD3L1 was not obvious at higher amounts of input DNA (Figure 2c, lanes 5-8), but it was essential to detect the methylated-CpG island at lower amounts of input DNA (Figure 2c, lanes 10 and 12). MBD3L1 protein could not provide methyl-CpG-binding ability for GST protein in a control reaction (Figure 2c, lane 4).

To further evaluate MIRA's sensitivity, we determined the minimal cell number that is essential for detecting a hypermethylated $R A S S F 1 A$ promoter (Figure 3). A few thousand cells were sufficient to detect the methylated-CpG island of the RASSF1A promoter. In all, $10^{3}$ cells were insufficient in this assay (Figure $3 \mathrm{a}$, lane 10 ), but $3 \times 10^{3}$ cells could provide a positive result (Figure $3 \mathrm{~b}$, lane 7 ). In subsequent assays, we routinely used $10^{4}$ cells per reaction and this number proved to be cell-type independent to provide sufficient amounts of input DNA (Figure 4).

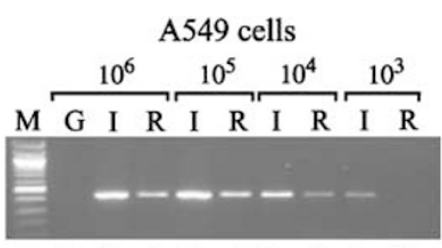

$\begin{array}{llllllllll}1 & 2 & 3 & 4 & 5 & 6 & 7 & 8 & 9 & 10\end{array}$

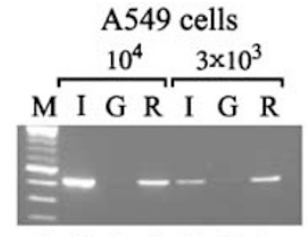

$\begin{array}{lllllll}1 & 2 & 3 & 4 & 5 & 6 & 7\end{array}$
Figure 3 MIRA of the RASSF1A promoter using small numbers of cells. Decreasing numbers of A549 cells were used in the assay. ' $G$ ' and ' $R$ ' refers to GST-only and GST-tagged MBD2b recovered samples. 'I' stands for input DNA.
To appraise MIRA's usability in the diagnosis of cancer-specific DNA hypermethylation, we studied the methylation status of the $R A S S F 1 A$ promoter in three additional cell lines (Figure 4). By analyzing different cell lines positive for RASSF1A promoter methylation (A549 and 267B1), we obtained results that exactly coincided with previous MSP analysis. ${ }^{21,24}$ We found that the RASSF1A promoter was unmethylated in the HPV-transformed Ki/HPV prostate epithelial cell line and in HeLa cells (Figure 4a, lane 10; Figure 4b, lane 4), as previously shown by bisulfite-based techniques. ${ }^{21,24}$ In the case of failed detection of a methylation-specific signal in a tested sample, we need to exclude the possibility that this was not because of loss of the input DNA during the procedure. Therefore, we performed PCR reactions using a LINE element-specific primer pair. LINE elements are retrotransposons inserted into the human genome in many copies $^{25}$ and are normally heavily methylated. ${ }^{26,27}$ Using this positive control, we can verify the unmethylated status of a CpG island of interest (Figure 4a, lane 11; Figure 4b, lane 5 ). These findings further confirm that the MIRA approach is suitable for monitoring the methylation status of specific regions of the genome. It is advisable to always include positive and negative controls (known methylated and unmethylated DNA samples), as well as a GST-only control.

To provide more data to strengthen the usability of MIRA, we tested the methylation pattern of the RASSF1A promoter in primary human tumor samples. In these experiments, blood DNA served as a negative (unmethylated) control. We found that the RASSF $1 A$ promoter was heavily methylated in pancreatic tumor samples, but not in the corresponding blood samples (Figure 4c). The same results for methylation of these samples were obtained previously by MSP. $^{28}$ Primary tumor samples often are mixtures of normal and cancer cells, making the detection more difficult. However, similar to MSP, MIRA is a positive detection assay for methylated DNA, and thus has the ability to detect methylated DNA even when the tumor cell population is outnumbered by normal cells in the sample or tissue. a

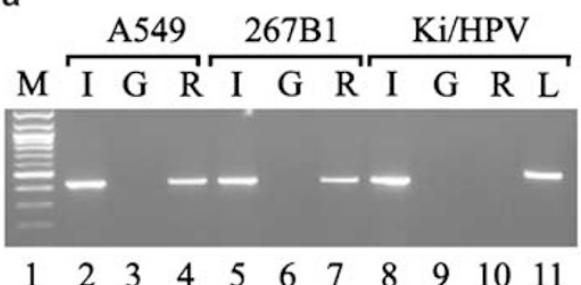

b

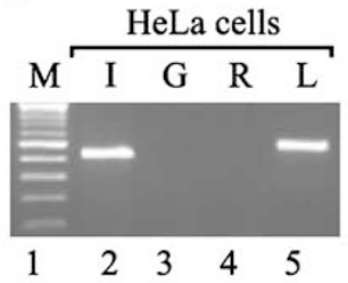

C

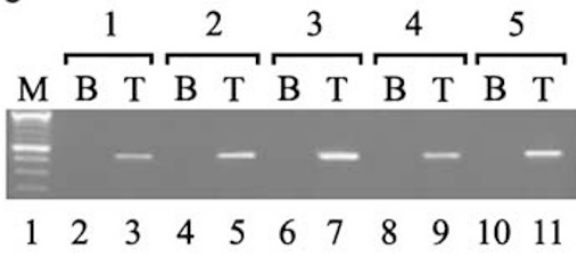

Figure 4 MIRA of the RASSF1A promoter using several cell lines and primary pancreatic cancer samples. (a, b) $10^{4}$ cells from different cell lines were used as starting material in these assays. ' $G$ ' and ' $R$ ' refer to GST only and GST-tagged MBD2b recovered samples, respectively. 'I' stands for input DNA. 'L' stands for LINE element-specific control. (c) Sonicated blood (15 ng) (b) or pancreatic tumor (T) DNA served as template for MIRA analysis. 
We next tested the binding capacity of the immobilized GST-MBD2b/MBD3L1 complex. We wanted to determine the lowest amount of input DNA, where no methylated DNA is detectable in the supernatant after performing a MIRA assay. First, we mixed different amounts of genomic DNA with a methylated RASSF1A promoter (from A549 cells) and unmethylated RASSF1A promoter (from HeLa cells) in such a way that the amount of input DNA was kept constant. Using the pre-mixed DNA, we performed the assay and treated the supernatant (unbound) and the pellet (bound) fractions with sodium bisulfite. The bisulfite-modified templates were analyzed by MSP (Figure 5a). We could not detect a methylated promoter signal in the supernatant fraction even in the sample with the highest input of methylated DNA (500 ng), while the pellet (bound) fraction was positive even in the case of the lowest input sample (125 ng). The interpretation of this result is that the MBD2b/MBD3L1 complex has a very high binding capacity and can deplete the supernatant from methylated templates. The input amount of GST-MBD2b protein was about $1 \mu \mathrm{g}$, which is equivalent to about $15 \mathrm{pmol}$ of protein, while the amount of the input DNA was in the femtomolar range. The result was further verified by bisulfite sequencing analysis (Figure 5b). The bisulfite-treated and PCR-amplified fragments from the supernatant and pellet fractions obtained from a mix of $50 \%$ unmethylated and $50 \%$ methylated DNA were cloned and sequenced. The amplified RASSF1A promoter fragment consists of 208 base pairs and contains 15 potential CpG methylation sites. The vast majority of these sites were methylated in clones isolated from the pellet fraction, while none of them was appreciably methylated in the supernatant fraction (Figure 5b). According to these data, the input MBD2b/MBD3L1 complex has a high and specific capacity to bind methylatedCpG-containing sequences.

The density of methylation may affect the binding affinity of MBD proteins, although, most often, the methylation of $\mathrm{CpG}$ islands, when it occurs in tumors, affects a large proportion of all CpG dinucleotides in a given CpG island. ${ }^{21}$ Although it has been demonstrated that MBD2 can recognize even a single methylated-CpG dinucleotide and neighboring nucleotide sequences have no effect on the binding ability, ${ }^{20}$ we next investigated how the density of methyl-CpGs influences the binding

a

Supernatant

Pellet

Supernatant

Pellet

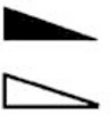

A549 DNA (methylated RASSF1A promoter)

HeLa DNA (unmethylated RASSF1A promoter)

\section{b}

\section{Isolated clones from supernatant}

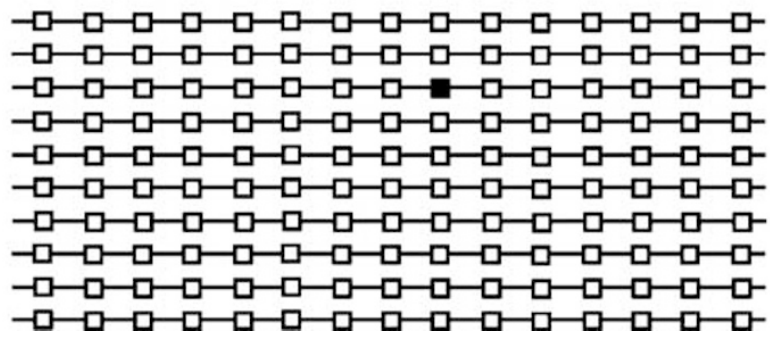

Isolated clones by MIRA

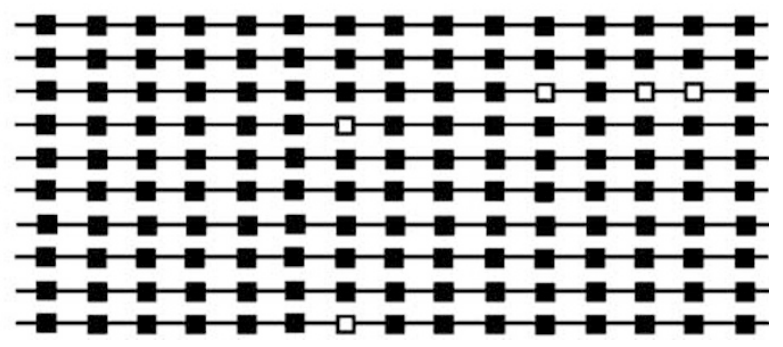

Figure 5 Binding capacity of the MIRA matrix. (a) The assay was performed on a constant amount of sonicated A549 and HeLa genomic DNA, and MSP was used to detect the methylation-specific signal. Lane 1, 500 ng of A549 DNA; lane 2, 375 ng A549 DNA and 125 ng of HeLa DNA; lane 3, $250 \mathrm{ng}$ A549 DNA and $250 \mathrm{ng}$ of HeLa DNA; lane 4, $125 \mathrm{ng}$ A549 DNA and 375 ng of HeLa DNA; lane 5, 500 ng of HeLa DNA. (b) Bisulfite sequencing. After performing MIRA, the DNA was isolated from the supernatant and the pellet fractions of a 1:1 mix of A549 and HeLa DNA (500 ng total), treated with sodium bisulfite, and subjected to PCR amplification with primers that target the RASSF1A promoter and have the capacity to amplify both methylated and unmethylated molecules. The amplified products were cloned and 10 clones each were sequenced. Open and filled squares indicate unmethylated and methylated-CpG dinucleotides, respectively. 
affinity of the MBD2b/MBD3L1 complex in MIRA. In order to determine how sensitive the method is, we prepared seven different fragments of the RASSF1A promoter region (Figure 6) in which one, two, three, five, 10 or 35 of its $\mathrm{CpG}$ dinucleotides were methylated. We observed that MIRA is sensitive enough to detect the methylated promoter when it contains only a single methylated-CpG dinucleotide. There were no detectable differences in MIRA's sensitivity using promoter fragments methylated at more than one position. One possible explanation is that the MBD2/MBD3L1 complex interacting with specific methyl-CpG dinucleotides may cover adjacent nucleotides and interfere with the binding of another MBD protein to that region. Therefore, the spacing between individual methylCpGs, and not their absolute number, can theoretically be a factor of MIRA's sensitivity. Practically, however, low-density methylation can clearly be detected (Figure 6).

Lastly, we extended our studies to the GSTP1 promoter to further prove MIRA's usability (Figure 7). Promoter methylation of the GSTP1 gene is a highly specific marker of prostate cancer. ${ }^{29-32}$ Genomic DNA was purified from prostate cancer samples and MIRA was performed on those templates and

a

RASSF1A promoter

GCCAAGTGTGTTGCTTCAGCAAACCGGACCAGGAGGGCCAGGGCCGGATGTGGGGACCC TCTTCCTCTAGCACAGTAAAGCTGGCCTCCAGAAACACGGGTATCTCCGCGTGGTGCTT TGCGGTCGCCGTCGTTGTGGCCGTCCGGGGTGGGGTGTGAGGAGGGGACGAAGGAGGGA AGGAAGGGCAAGGCGGGGGGGGCTCTGCGAGAGCGCGCCCAGCCCCGCCTTCGGGCCCC ACAGTCCCTGCACCCAGGTTTCCATTGCGCGGCTCTCCTCAGCTCCTTCCCGCCGCCCA GTCTGGATCCTGGGGGAGGCGCTGAAGTCGGGGCCCGCCCTGTGGCCCCGCCCGGCCCG CGCTTGCTAGCGCCCAAAGCCAGCGAAGCACGGGCCCAACCGGGCCATGTCGGGGGAGC CTGAGCTCATTGAGCTGCGGG

b RASSF1A promoter region

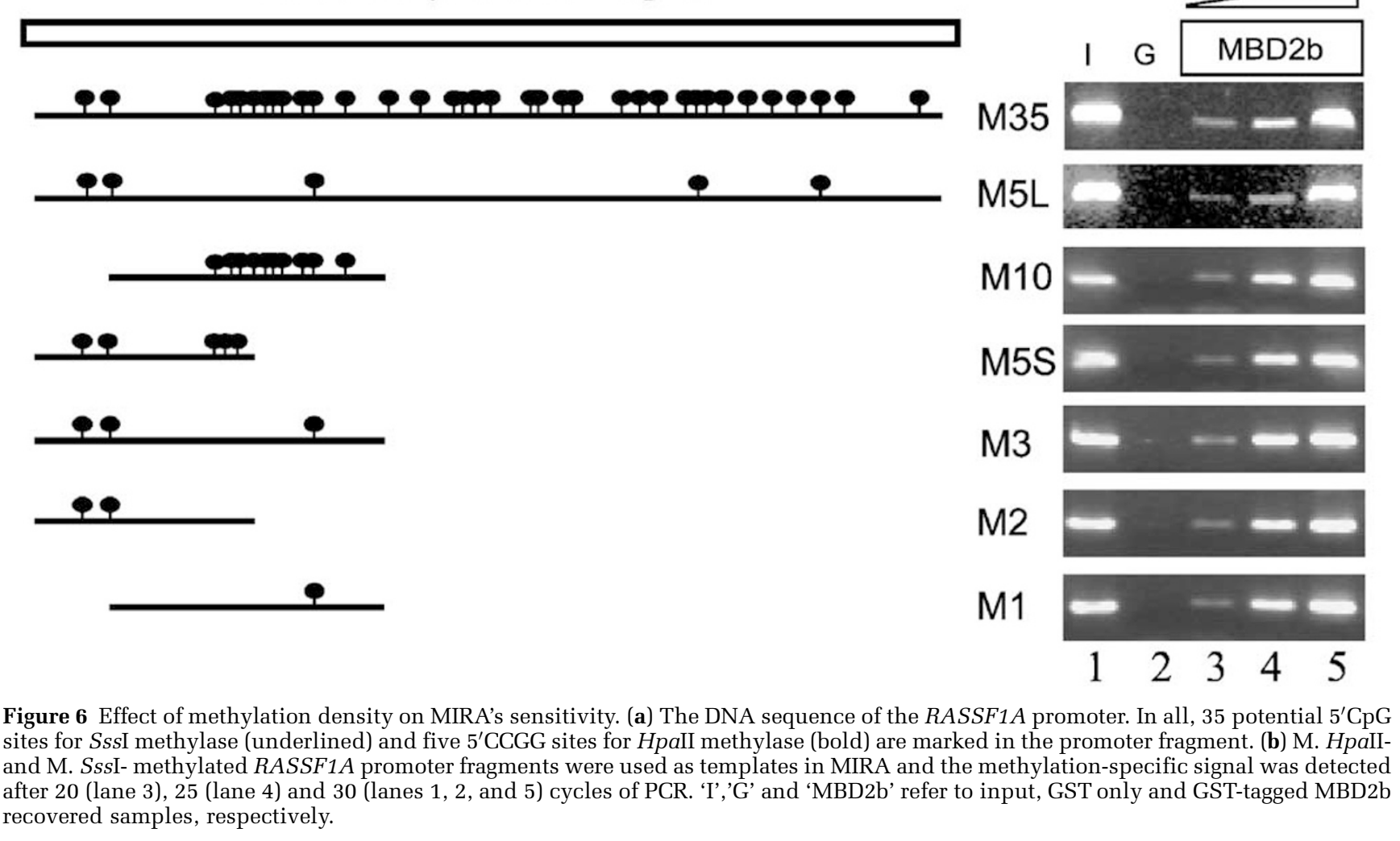




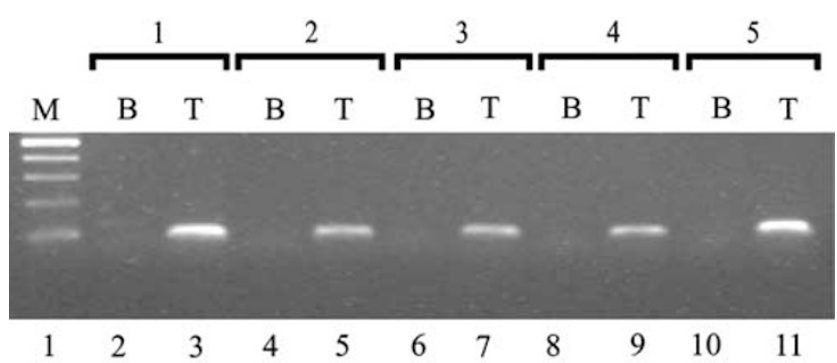

Figure 7 Analysis of GSTP1 promoter methylation by MIRA. MIRA of the GSTP1 promoter was performed on $15 \mathrm{ng}$ of sonicated blood (b) or prostate tumor (T) DNA samples.

blood DNA served as a negative control. Consistent with previous studies, our data verify that the GSTP1 promoter is frequently methylated in prostate cancer samples. According to the data obtained with the RASSF1A and GSTP1 promoters, we expect that MIRA can be used to analyze the methylation status of any $\mathrm{CpG}$ island.

The lowest detection limit of MSP is around $1 \mathrm{ng}$ of input DNA. ${ }^{17}$ The MIRA approach can also reach this sensitivity by adding MBD3L1 protein to the binding reaction (Figure 2c, lane 12). Bisulfite modification of DNA is a time-consuming procedure and the whole MSP approach takes about 2 days. After having the purified recombinant proteins available, the MIRA approach provides data within about $3 \mathrm{~h}$ (Figure 1). The purified and immobilized GST-MBD2b protein can be stored in buffer at $4^{\circ} \mathrm{C}$ for at least several weeks, while the His-tagged MBD3L1 protein can be kept in 50\% glycerolcontaining buffer at $-20^{\circ} \mathrm{C}$ for several months without losing its activity. The most time-consuming part of MIRA is the recombinant protein preparation, but after purifying reasonable amounts of the proteins the whole procedure is accomplished within only a few hours.

It is conceivable to replace the sonication step of the MIRA procedure with a restriction endonuclease digest that does not affect the $\mathrm{CpG}$ island sequences themselves. Endonucleases with AT-rich recognition sites such as MseI (5'TTAA) might be appropriate for this purpose. We also point out that this procedure essentially entails a substantial enrichment of genomic methylated-CpG island fractions. As such, this procedure may be adopted to microarray approaches in which the MIRA-enriched fraction is labeled for array hybridization.

The principle of MIRA is identical to MBD column chromatography, but its sensitivity is equivalent to MSP. In a clinical or diagnostic setting, MIRA may be applied for characterization of tumorspecific DNA methylation patterns in tumor-derived DNA itself, or in body fluids (serum, sputum, urine, etc) for early diagnosis of malignant disease. Since no ethanol precipitation steps are involved, the procedure may lend itself to automation to be used in routine diagnostic labs. In summary, MIRA is a specific and sensitive, but not laborious, approach that can be clinically useful in detection and diagnosis of any DNA methylation-related diseases, including cancer.

\section{Acknowledgements}

This work was supported by NIH grants CA88873 and CA104967 to GPP.

\section{References}

1 Bird AP. CpG-rich islands and the function of DNA methylation. Nature 1986;321:209-213.

2 Jones PA, Baylin SB. The fundamental role of epigenetic events in cancer. Nat Rev Genet 2002;3:415-428.

3 Costello JF, Fruhwald MC, Smiraglia DJ, et al. Aberrant CpG-island methylation has non-random and tumourtype-specific patterns. Nat Genet 2000;24:132-138.

4 Esteller M, Corn PG, Baylin SB, et al. A gene hypermethylation profile of human cancer. Cancer Res 2001;61:3225-3229.

5 Hendrich B, Bird A. Mammalian methyltransferases and methyl-CpG-binding domains: proteins involved in DNA methylation. Curr Top Microbiol Immunol 2000;249:55-74.

6 Wade PA. Methyl CpG binding proteins: coupling chromatin architecture to gene regulation. Oncogene 2001;20:3166-3173.

7 Hendrich B, Bird A. Identification and characterization of a family of mammalian methyl-CpG binding proteins. Mol Cell Biol 1998;18:6538-6547.

8 Jiang CL, Jin SG, Pfeifer GP. MBD3L1 is a transcriptional repressor that interacts with MBD2 and components of the NuRD complex. J Biol Chem 2004;279: 52456-52464.

9 Fraga MF, Esteller M. DNA methylation: a profile of methods and applications. Biotechniques 2002;33: 632-634, 636-649.

10 Shiraishi M, Oates AJ, Sekiya T. An overview of the analysis of DNA methylation in mammalian genomes. Biol Chem 2002;383:893-906.

11 Singer J, Roberts-Ems J, Riggs AD. Methylation of mouse liver DNA studied by means of the restriction enzymes msp I and hpa II. Science 1979;203:1019-1021.

12 Maxam AM, Gilbert W. Sequencing end-labeled DNA with base-specific chemical cleavages. Methods Enzymol 1980;65:499-560.

13 Pfeifer GP, Steigerwald SD, Mueller PR, et al. Genomic sequencing and methylation analysis by ligation mediated PCR. Science 1989;246:810-813.

14 Clark SJ, Harrison J, Paul CL, et al. High sensitivity mapping of methylated cytosines. Nucleic Acids Res 1994;22:2990-2997.

15 Frommer M, McDonald LE, Millar DS, et al. A genomic sequencing protocol that yields a positive display of 5-methylcytosine residues in individual DNA strands. Proc Natl Acad Sci USA 1992;89:1827-1831.

16 Xiong Z, Laird PW. COBRA: a sensitive and quantitative DNA methylation assay. Nucleic Acids Res 1997; 25:2532-2534.

17 Herman JG, Graff JR, Myohanen S, et al. Methylationspecific PCR: a novel PCR assay for methylation status of CpG islands. Proc Natl Acad Sci USA 1996;93:9821-9826. 
18 Cross SH, Charlton JA, Nan X, et al. Purification of CpG islands using a methylated DNA binding column. Nat Genet 1994;6:236-244.

19 Shiraishi M, Chuu YH, Sekiya T. Isolation of DNA fragments associated with methylated $\mathrm{CpG}$ islands in human adenocarcinomas of the lung using a methylated DNA binding column and denaturing gradient gel electrophoresis. Proc Natl Acad Sci USA 1999;96: 2913-2918.

20 Fraga MF, Ballestar E, Montoya G, et al. The affinity of different MBD proteins for a specific methylated locus depends on their intrinsic binding properties. Nucleic Acids Res 2003;31:1765-1774.

21 Dammann R, Li C, Yoon JH, et al. Epigenetic inactivation of a RAS association domain family protein from the lung tumour suppressor locus 3p21.3. Nat Genet 2000;25:315-319.

22 Dammann R, Schagdarsurengin U, Seidel C, et al. The tumor suppressor RASSF1A in human carcinogenesis: an update. Histol Histopathol 2005;20:645-663.

23 Jiang CL, Jin SG, Lee DH, et al. MBD3L1 and MBD3L2, two new proteins homologous to the methyl-CpGbinding proteins MBD2 and MBD3: characterization of MBD3L1 as a testis-specific transcriptional repressor. Genomics 2002;80:621-629.

24 Liu L, Zhang J, Bates S, et al. A methylation profile of in vitro immortalized human cell lines. Int J Oncol 2005;26:275-285.
25 Kazazian Jr HH, Goodier JL. LINE drive. retrotransposition and genome instability. Cell 2002;110:277-280.

26 Hata K, Sakaki Y. Identification of critical CpG sites for repression of L1 transcription by DNA methylation. Gene 1997;189:227-234.

27 Yang AS, Estecio MR, Doshi K, et al. A simple method for estimating global DNA methylation using bisulfite PCR of repetitive DNA elements. Nucleic Acids Res 2004;32:e38.

28 Dammann R, Schagdarsurengin U, Liu L, et al. Frequent RASSF1A promoter hypermethylation and K-ras mutations in pancreatic carcinoma. Oncogene 2003;22:3806-3812.

29 Yegnasubramanian S, Kowalski J, Gonzalgo ML, et al. Hypermethylation of $\mathrm{CpG}$ islands in primary and metastatic human prostate cancer. Cancer Res 2004; 64:1975-1986.

30 Lee WH, Morton RA, Epstein JI, et al. Cytidine methylation of regulatory sequences near the pi-class glutathione S-transferase gene accompanies human prostatic carcinogenesis. Proc Natl Acad Sci USA 1994;91:11733-11737.

31 Millar DS, Ow KK, Paul CL, et al. Detailed methylation analysis of the glutathione S-transferase pi (GSTP1) gene in prostate cancer. Oncogene 1999;18:1313-1324.

32 Cairns P, Esteller M, Herman JG, et al. Molecular detection of prostate cancer in urine by GSTP1 hypermethylation. Clin Cancer Res 2001;7:2727-2730. 